\title{
The QCD vacuum probed by overlap fermions
}

\author{
Volker Weinberg* \\ Deutsches Elektronen-Synchrotron DESY, 15738 Zeuthen, Germany \\ Institut für theoretische Physik, Freie Universität Berlin, 14196 Berlin, Germany \\ E-mail: volker.weinberg@desy.de
}

\section{Ernst-Michael Ilgenfritz}

Institut für Physik, Humboldt Universität zu Berlin, 12489 Berlin, Germany

E-mail: ilgenfriephysik.hu-berlin.de

\section{Karl Koller}

Sektion Physik, Universität München, 80333 München, Germany

E-mail: karl.kollerelrz.uni-muenchen.de

\section{Yoshiaki Koma}

Deutsches Elektronen-Synchrotron DESY, 22603 Hamburg, Germany

E-mail: yoshiaki.koma@desy.de

\section{Gerrit Schierholz}

Deutsches Elektronen-Synchrotron DESY, 22603 Hamburg, Germany

John von Neumann-Institut für Computing NIC, 15738 Zeuthen, Germany

E-mail: gerrit.schierholzddesy.de

\section{Thomas Streuer}

Department of Physics and Astronomy, University of Kentucky, Lexington, KY 40506-0055, USA

E-mail: streuerepa.uky.edu

\begin{abstract}
We summarize different uses of the eigenmodes of the Neuberger overlap operator for the analysis of the QCD vacuum, here applied to quenched configurations simulated by means of the Lüscher-Weisz action. We describe the localization and chiral properties of the lowest modes. The overlap-based topological charge density (with and without UV-filtering) is compared with the results of UV-filtering for the field strength tensor. The latter allows to identify domains of good (anti-)selfduality. All these techniques together lead to a dual picture of the vacuum, unifying the infrared instanton picture with the presence of singular defects co-existent at different scales.
\end{abstract}

XXIVth International Symposium on Lattice Field Theory

July 23-28, 2006

Tucson, Arizona, USA

\footnotetext{
*Speaker.
} 


\section{Introduction}

The understanding of the vacuum structure of QCD is an important part of theoretical particle physics. The distribution of the topological charge density is believed to describe nonperturbative phenomena like the large $\eta^{\prime}$ mass through the axial U(1) anomaly and the spontaneous breaking of chiral symmetry. The relation to confinement is less obvious. The instanton picture models the QCD vacuum as a random (quenched) or interacting (full QCD) instanton-antiinstanton system, whereas the understanding of confinement seems to require singular fields emanating from lowerdimensional defects [1].

Recent advances in implementing chiral symmetry on the lattice have made it possible to find, from first principles, manifestations of these dual aspects of vacuum structure. Overlap fermions are described by the Neuberger-Dirac operator $D_{N}$ constructed as a solution of the Ginsparg-Wilson relation. They are the cleanest known implementation of lattice fermions. For gauge fields free of dislocations, the index theorem is unambiguously realized and the spectrum consists of chiral zero modes and non-chiral non-zero modes. While the number and chirality of zero modes provides a value for the total topological charge, the non-chiral non-zero modes appear in pairs of eigenvalues $\pm i \lambda$.

For our methodical study we use quenched configurations simulated by means of the LüscherWeisz action. In the following table we list the statistics of lattices used in our investigation. We show also the topological susceptibility $\chi_{t}$. To set the scale, we use the quenched pion decay constant $f_{\pi}=91(2) \mathrm{MeV}$.

\begin{tabular}{|c|c|c|c|r|r|r|c|}
\hline$\beta$ & $a[\mathrm{fm}]$ & lattice size & $L[\mathrm{fm}]$ & $V\left[\mathrm{fm}^{4}\right]$ & $\chi_{t}\left[\mathrm{fm}^{-4}\right]$ & confs. & modes \\
\hline \hline 8.45 & $0.105(2)$ & $12^{3} \times 24$ & 1.26 & 5.04 & $0.517(38)$ & 437 & 50 \\
8.45 & $0.105(2)$ & $16^{3} \times 32$ & 1.68 & 15.93 & $0.533(38)$ & 400 & $136-151$ \\
8.45 & $0.105(2)$ & $24^{3} \times 48$ & 2.52 & 80.66 & $0.522(48)$ & 250 & $144-177$ \\
\hline 8.10 & $0.138(3)$ & $12^{3} \times 24$ & 1.66 & 15.04 & $0.647(58)$ & 251 & $137-154$ \\
\hline 8.00 & $0.154(4)$ & $16^{3} \times 32$ & 2.46 & 73.72 & $0.607(21)$ & 2156 & $160-178$ \\
\hline
\end{tabular}

After presenting results concerning localization and chiral properties of individual eigenmodes in different parts of the spectrum up to $\lambda=400 \mathrm{MeV}$, we describe two specific applications of the overlap Dirac operator. One is aiming to extract a UV-filtered field strength tensor [2] in order to assess the local degree of (anti-)selfduality. The other uses the fermionic definition of topological charge density [3]. We interpret the respective structures and their dimensionality.

\section{Localization, dimensionality and local chirality of the lowest eigenmodes}

Recently, the localization, the eventually lower dimensionality and the local chirality of the lowest eigenmodes of the Dirac operator have attracted a lot of interest [4, 5]. The reason for this activity is that these modes might be pinned down by some topological defects. This is inspired by certain confinement mechanisms, e.g. the center vortex mechanism. In model field configurations of thick vortices the zero modes have been seen [6] localized along the vortices with additional peaks at the intersections. The particular interest in the lowest eigenmodes is motivated by the 
observation that the lightest hadron propagators are very well approximated by taking only $O(50)$ lowest eigenmodes in the quark propagator into account.

A useful measure to quantify the localization of eigenmodes is the inverse participation ratio (IPR), $I=V \sum_{x} \rho(x)^{2}$, with the scalar density $\rho(x)=\psi_{\lambda}^{\dagger}(x) \psi_{\lambda}(x)$ for eigenfunctions with eigenvalues $i \lambda$, which are conventionally normalized as $\sum_{x} \rho(x)=1$. In Fig.1 (a) we plot the IPR averaged over bins with a bin width $\Delta \lambda=50 \mathrm{MeV}$ and for the zero modes considered separately. The average IPR shows an $L$ and $a$ dependence for zero modes and for non-zero modes only in the range $\lambda<150 \mathrm{MeV}$. Beyond that the average IPR corresponds to 4-dimensionally extended states. In the theory of the metal-insulator transition, a more detailed description of the localization properties at different "heights" of the wave functions has led to consider generalized IPRs like $I_{p}=\sum_{x} \rho(x)^{p}$, averaged over parts of the spectrum and different realizations of disorder. In any physical application, dimensionalities $d^{*}(p)$ different from the embedding dimension $d$ and eventually varying with $p$, can be inferred from the volume scaling,

$$
\begin{array}{lll}
I_{p} \propto L^{-d(p-1)} & \text { metallic phase, } & \text { extended wave functions }, \\
I_{p} \propto L^{-d^{*}(p)(p-1)} & \text { critical region, } & \text { multifractal wave functions }, \\
I_{p} \propto \text { const } & \text { insulator phase, } & \text { localized wave functions }
\end{array}
$$

While the dimension for the metallic or insulator phase is $d$ or 0 , respectively, the critical multifractal region in between is characterized by a multitude of fractal dimensions $d^{*}(p)$ and also by critical level statistics [7].

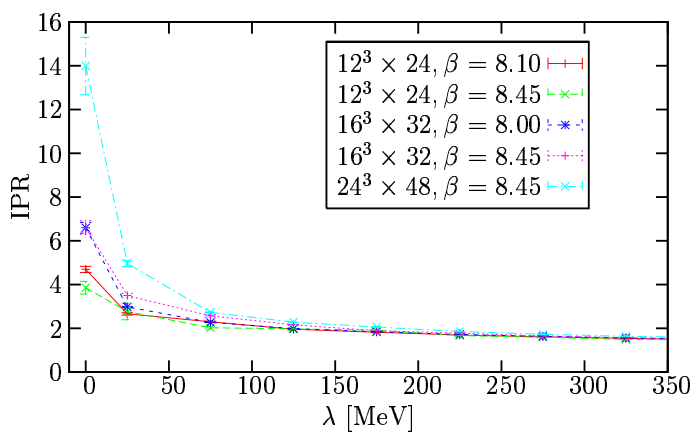

(a)

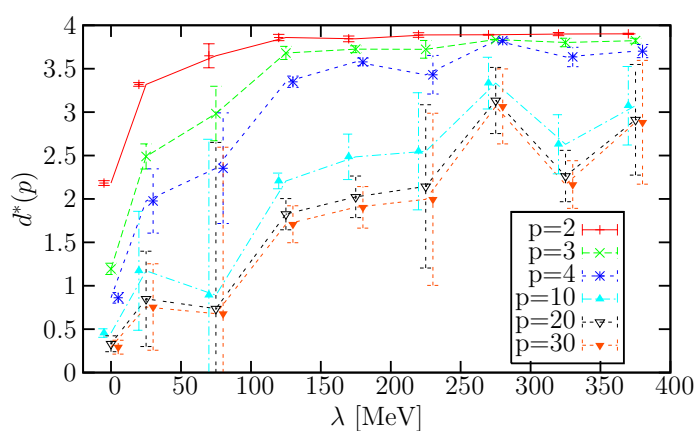

(b)

Figure 1: (a) The average IPR is shown for zero modes and for non-zero modes in $\lambda$ bins of width $50 \mathrm{MeV}$ for the five ensembles. (b) The fractal dimensionality $d^{*}(p)$ obtained from fits of the volume dependence of the averages of the generalized $I_{p}$ is presented for zero modes and for non-zero modes in $\lambda$ bins of width $50 \mathrm{MeV}$ for the three ensembles with different volumes and common $\beta=8.45$.

From a fit of the volume dependence of $I_{p}$ we find that all eigenstates with $\lambda<200 \mathrm{MeV}$ in this sense belong to a critical regime. From the standard IPR, $I=V I_{2}$, we conclude that the dimensionality of the zero modes is close to two. In the next bins of width $50 \mathrm{MeV}$ each, the dimensionality is near three, in agreement with [4] and disagreement with [5] ${ }^{1}$. The IPR of the

\footnotetext{
${ }^{1}$ However, in [4] the Asqtad Dirac operator on quenched Symanzik improved gauge fields is used, while the analysis in [5] is done with overlap fermions on quenched SU(2) configurations generated with the standard Wilson action.
} 
higher modes is independent of $V$ and $a$, describing the observation that the modes freely extend throughout $d^{*}(2) \lesssim d=4$ dimensions.

Looking at the higher moments of IPR one can see a decrease of the multifractal dimension with increasing $p$ as shown in Fig. 1 (b). This reflects the fact that $I_{p}$ with $p>2$ explores regions of higher scalar density. For $p=20 \ldots 30$ the emerging fractal dimension seems to converge. The envelope reveals that the maxima of the scalar density of zero modes and the lowest non-zero modes up to $\lambda=100 \mathrm{MeV}$ are characterized by $d^{*}(p)<1$ localization (pointlike peaks), whereas in the spectral region $100 \mathrm{MeV}<\lambda<400 \mathrm{MeV}, d^{*}(p)=2$ or 3 characterizes the maxima of the scalar density. In contrast to this, the standard IPR alone would describe the modes in the spectral region $\lambda>150 \mathrm{MeV}$ as four-dimensional. For the zero modes, the results favor an interpretation in the vortex picture above, but that needs direct confirmation.

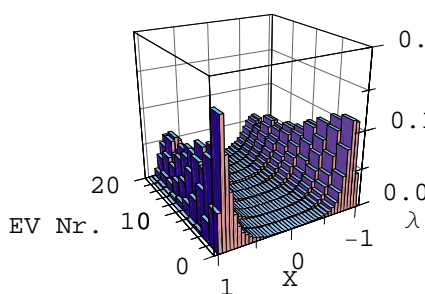

(a)

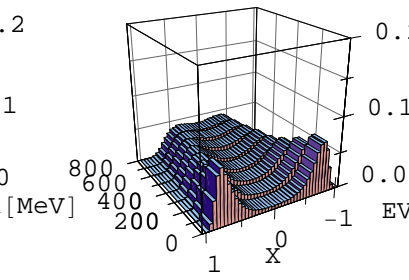

(b)

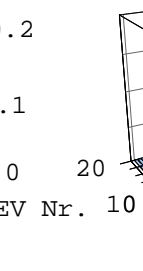

10

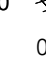

(c)

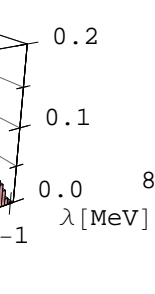

c)

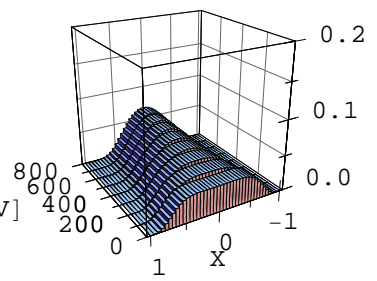

(d)

Figure 2: Normalized histograms of the local chirality $X(x)$ in the $Q=0$ subsample (consisting of 37 configurations) among the $16^{3} \times 32$ lattices generated at $\beta=8.45$. (a) and (c): for the lowest ten pairs (with positive and negative $\lambda$ ) averaged over the subsample. (b) and (d): for all modes from the subsample, averaged over eight $\lambda$ bins of width $100 \mathrm{MeV}$. (a) and (b): with a cut for $1 / 100$ of the sites with biggest scalar density $\rho(x)$. (c) and (d): with a cut for $1 / 2$ of the sites.

The local chiral density $\rho_{\lambda 5}(x)=\psi_{\lambda}^{\dagger}(x) \gamma_{5} \psi_{\lambda}(x)$ with $\sum_{x} \rho_{\lambda 5}(x)= \pm 1$ or 0 for chiral or nonchiral modes, respectively, is equally important. Whereas the zero modes are entirely chiral, the non-zero modes with globally vanishing chirality may still have a rich local chirality structure correlated with the local (anti-)selfduality of the background gauge field. A measure proposed by Horvath [8] to describe the local chirality $\rho_{\lambda \pm}(x)=\psi_{\lambda}^{\dagger}(x) P_{ \pm} \psi_{\lambda}(x)$ (with projectors $P_{ \pm}=\left(1 \pm \gamma_{5}\right) / 2$ onto positive/negative chirality) is given by

$$
X(x)=\frac{4}{\pi} \arctan \left(r_{\lambda}(x)\right)-1 \in[-1,+1],
$$

with $r_{\lambda(x)}=\rho_{\lambda+}(x) / \rho_{\lambda-}(x)$. For the chiral zero modes $X(x) \equiv \pm 1$, while for the non-chiral nonzero modes $X(x)$ is a strongly varying field.

In Fig. 2 normalized histograms with respect to $X(x)$ are shown for the $Q=0$ subsample (37 configurations) of the $16^{3} \times 32$ lattices generated at $\beta=8.45$, with cuts including $1 / 100$ and $1 / 2$ of the sites according to the biggest $\rho(x)$. For zero modes, the histograms would be concentrated at $X= \pm 1$ irrespective of any cut. For the non-zero modes, it turns out that with a more restrictive cut in $\rho(x)$ the local chirality, i.e. the concentration of the histogram over the included lattice points, can be driven towards $X= \pm 1$. A similar enhancement can be seen towards the lower modes. For the lowest two pairs of non-zero modes all sites with $\rho(x)$ above median show the two chiral peaks. 


\section{The UV-filtered gluonic field strength tensor and the topological charge density}

The lowest eigenmodes of the overlap operator can directly be used as a filter of the underlying gauge fields. Following a method proposed by Gattringer [2], the gluonic field strength tensor can be represented as

$$
F_{\mu v}^{a}(x) \propto \sum_{j} \lambda_{j}^{2} f_{\mu v}^{a}(x)_{j} .
$$

The contribution of the $\mathrm{j}$-th eigenmode $f_{\mu \nu}^{a}(x)_{j}=-\frac{i}{2} \psi_{j}^{\dagger}(x) \gamma_{\mu} \gamma_{\nu} T^{a} \psi_{j}(x)$ to the field strength tensor $F_{\mu \nu}^{a}(x)$ is projected out by means of an appropriate combination of $\gamma$-matrices with the SU(3) generator $T^{a}$. To study the local (anti-)selfduality of the filtered field strength tensor, we analyze the ratio

$$
r(x)=(\tilde{s}(x)-\tilde{q}(x)) /(\tilde{s}(x)+\tilde{q}(x)),
$$

with the action density $\tilde{s}(x)=\operatorname{Tr} F_{\mu \nu}(x) F_{\mu \nu}(x)$ and the topological density $\tilde{q}(x)=\operatorname{Tr} F_{\mu \nu}(x) \tilde{F}_{\mu \nu}(x)$ of the filtered field strength summing in Eq. (3.1) over different sets of low-lying non-zero modes. Using the ratio $r(x)$ we do not need to know the normalization factor of $\tilde{s}(x)$ and $\tilde{q}(x)$.

Mapping $r(x)$ to $R(x) \in[-1,+1]$ analogously to Eq. (2.1), one would get $R(x) \equiv \pm 1$ for totally (anti-)selfdual fields. In Fig. 3 we present histograms with respect to $R(x)$ applying two different cuts with respect to the filtered action density $\tilde{s}(x)$. We observe peaks at $R= \pm 1$ that become weaker with the inclusion of more modes. The peaks become more pronounced again when one concentrates on lattice points above some cut with respect to the filtered action density $\tilde{s}(x)$.

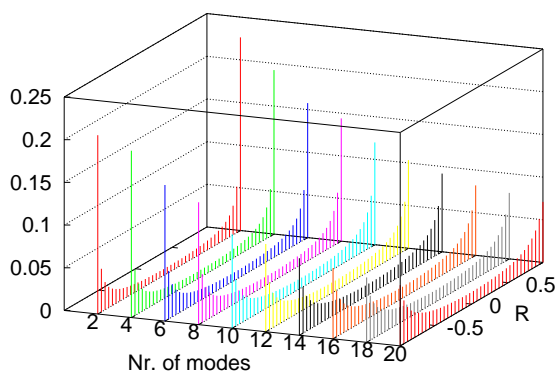

(a)

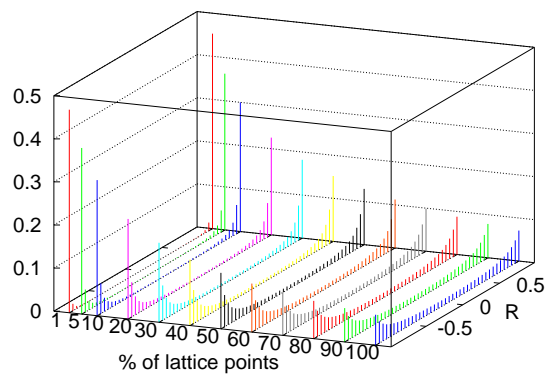

(b)

Figure 3: Normalized histograms with respect to the local (anti-)selfduality $R(x)$ of the field strength tensor in the $Q=0$ subsample of 37 configurations generated on the $16^{3} \times 32$ lattice at $\beta=8.45$, (a) taken over the full volume, but depending on the number of non-zero modes (2 - 20) included in the filter, (b) applying various cuts according to the action density for a fixed number (20) of eigenmodes included in the filter.

Alternatively to $\tilde{q}(x)$ based on the filtered field strength tensor we also examine the topological charge density as given by the formula $q(x)=-\operatorname{Tr}\left[\gamma_{5}\left(1-\frac{a}{2} D_{N}(x, x)\right)\right]$ [3] which holds for any $\gamma_{5}$-Hermitean Dirac operator satisfying the Ginsparg-Wilson relation. We use it in a twofold way [9, 10]. (i) We directly calculate the trace of the overlap operator. The "all-scale" density $q(x)$ computed in this numerically very expensive way includes charge fluctuations at 
all scales down to the lattice spacing $a$. (ii) We use the mode-truncated spectral representation $q_{\lambda_{\text {cut }}}(x)=-\sum_{|\lambda|<\lambda_{\text {cut }}}\left(1-\frac{\lambda}{2}\right) \rho_{\lambda 5}(x)$.

In Ref. [11] we have begun a cluster analysis of the two forms of the topological density. The number of separable clusters of $q(x)$ grows without limit with $a \rightarrow 0$, and similarly the number of clusters of $q_{\lambda_{\text {cut }}}$ with increasing $\lambda_{\text {cut }}$ at fixed $a$. We have also seen [12] that the negativity of the two-point-function of the topological charge density [13] (up to a positive core due to the nonultralocality of $q(x)$ ) is realized for the all-scale density only and increases with decreasing lattice spacing $a$. The correlator for $q_{\lambda_{\text {cut }}}$ describes clusters of the mode-truncated density. For sufficiently large $\lambda_{\text {cut }}$, clusters of different sign are attractively correlated as demonstrated by a negative tail of the corresponding correlation function.

In Fig. 4 (a)-(c) we compare in a two dimensional cut the density $q \lambda_{\text {cut }}$ with $\lambda_{\text {cut }}=200 \mathrm{MeV}$, the density $\tilde{q}(x)$ from the filtered field strength tensor and the all-scale density $q(x)$. Viewed is a typical configuration generated at $\beta=8.45$ on a $16^{3} \times 32$ lattice. Normalizing $\tilde{q}(x)$ in such a way that the maxima of $\tilde{q}(x)$ and $q_{\lambda_{\text {cut }}}(x)$ are equal, we see that the two definitions (both including 18 modes) are in very good agreement. The density of clusters is $O\left(1 \mathrm{fm}^{-4}\right)$, and the number of modes necessary to see the clusters approximately corresponds to the number of clusters. The topological susceptibility is smaller by a factor of $1 / 2$ compared to the dilute instanton-gas approximation. This is an independent indication for pairing correlations among oppositely charged clusters of $q \lambda_{\text {cut }}$.

In contrast the cut through the all-scale topological density seems to be completely random. It is interesting to notice that structure can be extracted from this "noise" as well [14, 11]. First of all, cluster percolation at low $q(x)$ leads to the two thin (thickness of order $O(a)$ ) sign-coherent global clusters for the first time seen by Horvath et al. [10]. At higher $|q(x)|$, exploring the interior of the clusters leads to the result that the topological landscape is also multifractal, with fractal dimensions ranging from $d^{*}=0.7$ to $d^{*}=2.6$.

Finally, Fig. 4 (d) and (e) show the positive and negative part of the local (anti-)selfduality variable $R(x)$. One can see that the peaks of the mode-truncated topological density coincide with the domains of (anti-)selfduality. This is evidence that with the chosen smearing parameters, extended lumps of high topological charge density may be interpreted as (anti-)selfdual objects. On the other hand, the all-scale density allows one simultaneously to localize singular fields with fractal dimension $d^{*}<4$. A more quantitative analysis based on our cluster algorithm as drafted in [11] will be discussed in detail in a forthcoming publication.

\section{Acknowledgements}

The numerical calculations have been performed on the IBM p690 at HLRN (Berlin) and NIC (Jülich), as well as on the PC farms at DESY Zeuthen, LRZ Munich and the University of Munich. We thank these institutions for support. Part of this work is supported by DFG under contract FOR 465 (Forschergruppe Gitter-Hadronen Phänomenologie). 


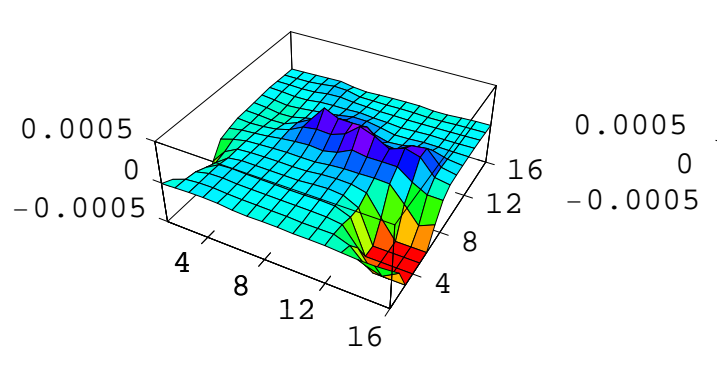

(a)

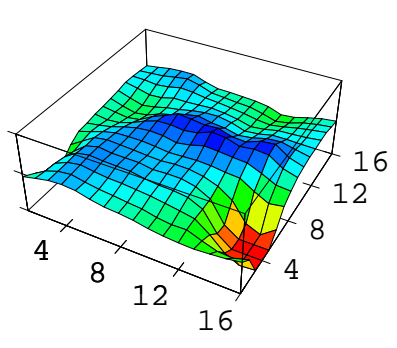

(b)

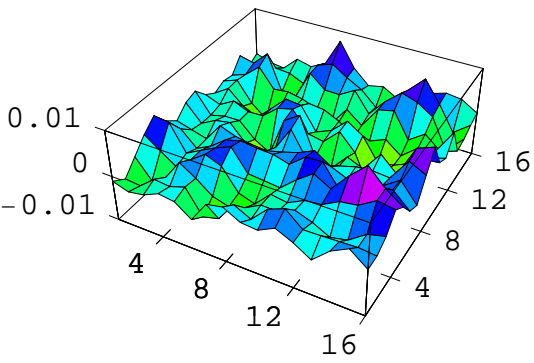

(c)

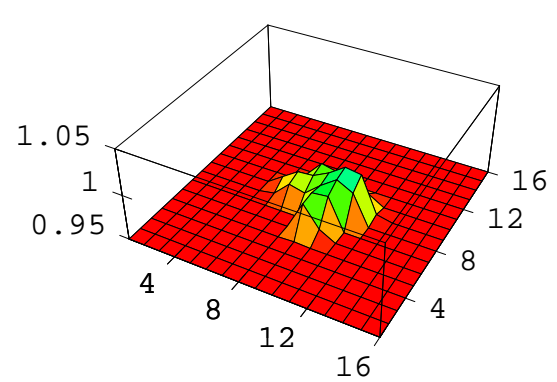

(d)

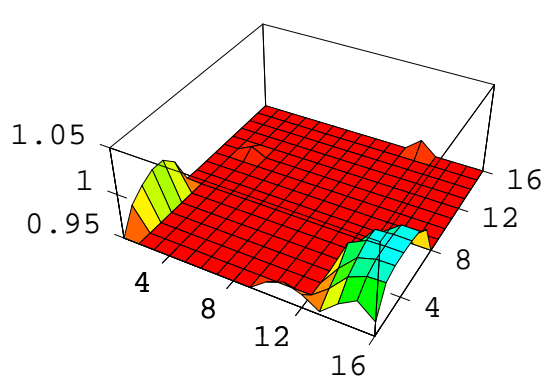

(e)

Figure 4: Two-dimensional cuts through a typical configuration generated at $\beta=8.45$ on a $16^{3} \times 32$ lattice. Direct comparison of (a) $\tilde{q}(x)$ based on the filtered field strength tensor, (b) $q_{\lambda_{\text {cut }}}(x)$ as the mode-truncated density and (c) the all-scale charge density $q(x)$. Subpanels (d) and (e) show the positive and negative part of the local (anti-)selfduality variable $R(x)$. Except for (c) always 18 eigenmodes are taken into account.

\section{References}

[1] V. I. Zakharov, Phys. Atom. Nucl. 68, 573 (2005), Yad. Fiz. 68, 603 (2005), [hep-ph/0410034].

[2] C. Gattringer, Phys. Rev. Lett. 88, 221601 (2002), [hep-lat/0202002].

[3] P. Hasenfratz, V. Laliena, and F. Niedermayer, Phys. Lett. B427, 125 (1998), [hep-lat/9801021].

[4] C. Aubin et al., Nucl. Phys. Proc. Suppl. 140, 626 (2005) [hep-lat/0410024],

C. Bernard et al., [PoS(LAT2005)299].

[5] F. V. Gubarev et al., JETP Lett. 82, 343 (2005), |hep-lat/0505016].

[6] J. Gattnar et al., Nucl. Phys. B716, 105 (2005), hhep-lat/0412032].

[7] V. E. Kravtsov, [cond-mat/9603166].

[8] I. Horvath et al., Phys. Rev. D65, 014502 (2002), [hep-lat/0102003].

[9] I. Horvath et al., Phys. Rev. D67, 011501 (2003), [hep-lat/0203027].

[10] I. Horvath et al., Phys. Rev. D68, 114505 (2003), [hep-lat/0302009].

[11] E.-M. Ilgenfritz et al., Nucl. Phys. Proc. Suppl. 153, 328 (2006), [hep-lat/0512005].

[12] Y. Koma et al., [PoS(LAT2005)300].

[13] I. Horvath et al., Phys. Lett. B617, 49 (2005), |hep-lat/0504005].

[14] I. Horvath et al., Phys. Lett. B612, 21 (2005), |hep-lat/0501025]. 